\title{
Clinicopathological Predictive Factors of Melanoma Lung Metastases
}

Giovanni Paolino ${ }^{3 *}$, Ugo Bottoni ${ }^{2}$, Rita Clerico ${ }^{3}$, Dario Didona ${ }^{3}$, Federico Venuta ${ }^{1}$, Paola Corsetti ${ }^{3}$, Marina Ambrifi ${ }^{3}$, Carmen Cantisani ${ }^{3}$, Antonio Giovanni Richetta $^{3}$, Teresa Lopez ${ }^{3}$ and Stefano Calvieri ${ }^{3}$

${ }^{1}$ Department of Thoracic Surgery, La Sapienza University of Rome, Viale del Policlinico 155, 00186 Rome, Italy

2University Magna Graecia, V.le Europa, 88100 Catanzaro, Italy

${ }^{3}$ Dermatologic Clinic, La Sapienza University of Rome, Viale del Policlinico 155, 00186 Rome, Italy

*Corresponding author: Paolino G, La Sapienza University of Rome, Viale del Policlinico 15, 00186 Rome, Italy, Tel: +39 3498465167 ; Fax: +39 064462104 ; E-mail: paolgio@libero.it

Rec date: May 12, 2014, Acc date: June 2, 2014, Pub date: June 12, 2014

Copyright: (c) 2014 Paolino G, et al. This is an open-access article distributed under the terms of the Creative Commons Attribution License, which permits unrestricted use, distribution, and reproduction in any medium, provided the original author and source are credited.

\section{Abstract}

Background: The lung is the second most common site for metastatic malignant melanoma, with a poor prognosis. In this regard, identify clinicopathological predictors for Melanoma Lung Metastases (MLM) plays a pivotal role in clinical practice.

Methods: We computer-searched the clinical records of all our patients registered in our melanoma database to identify patients that presented MLM. Kaplan-Meier product was used to estimate time to MELANOMA LUNG METASTASES (TMLM) and Overall Survival (OS); while the log-rank test was used to evaluate differences between the survival curves. Cox proportional hazards regression was performed in the analysis between clinicopathological features of the primary tumor and MLM.

Results: A total of 63 patients with MLM were included in our analysis. Median TMLM was 27.4 months, while median OS was 55.5 months, with a Median Lung Metastases Survival (MLMS) of 10 months. Melanoma patients with a primary axial tumor $(p<0.001)$ and with an age $\leq 60$ years $(p=0.01)$ showed a better TMLM. While OS was statistically significant higher only in axial melanomas $(p<0.001)$, multivariate analysis showed that peripheral site of the primary tumor remained the main predictor to develop MLM, with a significant influence in TMLM and also in the long-term $(p<0.01$ and $p=0.04)$.

Conclusions: Currently no standardized therapies exist for MLM. In this regard, the prevention of secondary recurrences plays a pivotal role in the management of melanoma patients. According to our results, peripheral melanoma is the main predictor for development of MLM.

Keywords: Melanoma; Lung metastases; Survival; Predictive factors

\section{Introduction}

The lungs are the second most frequent site of metastases from extra thoracic malignancies, while $20 \%$ of metastatic diseases is isolated to the lungs [1]. At the same time, they are the second most common site for metastatic malignant melanoma, with a one-year survival rate of $53 \%$ [1].

Metastasectomy remains the primary treatment for Melanoma Lung Metastases (MLM) with $\leq 2$ nodules and chemotherapy is principally used in those with multiple metastases. Several chemotherapeutic drugs are available, including dacarbazine, cisplatin, carmusine (also in combination with various immunotherapies), BOLD-regimen+G-CSF, ipilimumab, nivolumab, vemurafenib and the association between dabrafenib and trametenib.

However, although different authors have identified clinical variables present at time of MLM diagnosis as predictive of post-MLM survival, relatively few studies have been conducted to identify patients with increased risk of developing MLM, basing on the characteristics of the primary tumour. In this regard, factors such as site of primary tumour, Breslow thickness, ulceration, and Sentinel Lymph Node
(SLN) can play an important role as predictors for a secondary pulmonary localization and for a relative better management of the patient.

We conducted a study of our patients with MLM, in order to identify risk factors useful to predict the development of MLM and to evaluate the impact of the clinicopathological features of the primary tumour on time to Melanoma Lung Metastases (TMLM) and Overall Survival (OS).

\section{Material and Methods}

We computer-searched the clinical records of all our patients registered in our melanoma database from June 1998 to June 2013, to identify patients that presented or developed Melanoma Lung Metastases (MLM).

Clinical and pathological data were obtained from the electronic database. The following parameters were registered: demographics, anatomical localization and histological characteristics of primary melanoma, time interval between diagnosis of primary melanoma and first recurrence, time interval between diagnosis of primary melanoma and MLM, localization of other visceral metastases and date of death and/or last follow-up. 
Page 2 of 5

The diagnosis of MLM was usually obtained by chest radiography, computed tomography and thoracothamy. If possible a histological examination was performed. MLM diagnosed within 30 days of diagnosis of the primary melanoma were considered as synchronous.

We followed all patients from the date of the first visit to date of death or last follow-up. Patients underwent regular follow-up in our Department with periodical exams and instrumental assessment through radiography, sonography, CT, MRI, bone scintigraphy and PET.

To establish risk factors we analyzed the following parameters: sex (female or male), age ( $\leq 60$ or $\geq 61$ years), anatomic site of primary tumor (axial or peripheral), Breslow thickness $(\leq 1.0 \mathrm{~mm}$ or $\geq 1.01$ $\mathrm{mm}$ ), and ulceration (presence or absence), and sentinel lymph node biopsy (positive or negative). For anatomic sites of primary tumor, axial site included head, neck and trunk, while peripheral sites included upper and lower limbs. These parameters are the same used by Balch et al. to establish AJCC melanoma staging $7^{\text {th }}$ edition [2].

\section{Statistical analysis}

The Incidence Rate (IR) was calculated as the ratio between the observed patients with MLM and all melanoma patients founded in our electronic database. Then we analyzed the following parameters: Melanoma Lung Metastases Survival (MLMS), Disease Free Survival (DFS), time to Melanoma Lung Metastases (TMLM) and Overall Survival (OS). DFS was calculated from diagnosis of primary tumor to first recurrence, while TMLM from melanoma diagnosis to MLM. Assuming that the effects of the predictor variables are constant over time, we used Cox proportional hazards regression between MLM and the characteristics of disease at the time of first diagnosis. MLMS was calculated from diagnosis of MLM to death and/or last follow-up, while OS was calculated from first melanoma diagnosis to death and/or last follow-up. Kaplan-Meier product was used to estimate curves for TMLM and OS, and the log-rank test was used to evaluate differences between the survival curves. Patients who were lost to follow-up or who were alive at the time of last follow-up were censored at the date of their last follow-up. A $p$ value $<0.05$ was considered statistically significant.

\section{Results}

A total of 63 patients were included in the analysis. They were 21 female (33\%) and 42 male (67\%), with an IR equal to 1:29 melanoma patients present in our melanoma database. The methods of diagnosis were chest radiography $(n=27)$, computed tomography $(n=25)$ and thoracotomy $(n=11)$. The median age at time of lung metastases was of 55.5 years (ranging $22-78$ years). Twenty-four were $\geq 61$ years while 39 were $\leq 60$ years (Table 1 ).

Regarding the first diagnosis, 59 patients showed a primary cutaneous melanoma and 1 patient a mucosal melanoma. The anatomic site of the primary tumor was axial in 40 patients and peripheral in 19 patients. The Sentinel Lymph Node (SNL) biopsy was positive in 10 patients and negative in 50 patients; two patients presented an occult nodal melanoma while 1 patient a primary melanoma of the lung (melanoma with unknown primary).

The Breslow thickness was $\geq 1.01 \mathrm{~mm}$ in 52 patients (88\%), with a median thickness of $3.75 \mathrm{~mm}$ (ranging $1.01 \mathrm{~mm}-18 \mathrm{~mm}$ ); while it was $\leq 1.0 \mathrm{~mm}$ only in 7 patients (12\%) with a median thickness of $0.65 \mathrm{~mm}$ (ranging $0.6 \mathrm{~mm}-0.9 \mathrm{~mm}$ ). In one patient with mucosal melanoma,
Breslow thickness was not evaluated. The ulceration of the primary tumor was present in 18 patients (30.5\%) (Table 1).

\begin{tabular}{|l|l|l|}
\hline & Number $(\mathbf{n})$ & Percentage (\%) \\
\hline Gender & & \\
\hline Male & 42 & 67 \\
\hline Female & 21 & 33 \\
\hline Age & & \\
\hline$\leq 60$ (17 M : 22 F) & 39 & 62 \\
\hline$\geq 60$ (20 M : 4 F) & 24 & 38 \\
\hline Cutaneous Sites & & \\
\hline Peripheral & 19 & 32 \\
\hline Axial & 40 & 68 \\
\hline Breslow & & \\
\hline$\leq 1.00$ mm & 52 & 88 \\
\hline$\geq 1.1$ mm & 7 & 12 \\
\hline Ulceration* & 10 & 30 \\
\hline Presence & 50 & 70 \\
\hline Absence & 41 & \\
\hline SLN* & & 37 \\
\hline Positive & & \\
\hline Negative & & \\
\hline * excluding melanoma with unknown primary and mucosal melanoma; \\
$* *$ excluding melanoma with unknown primary. & \\
\hline
\end{tabular}

Table 1: Baseline and primary tumour characteristics of melanoma patients who developed lung metastases

At the time of melanoma lung metastases (MLM) diagnosis, 26 patients (41\%) presented also other secondary visceral lesions, while the remaining 39 patients presented no other visceral involvement. The extra pulmonary sites of metastases were nodal (64\%) cutaneous and subcutaneous tissues (20\%), bone (4\%), ovary (4\%) and brain (8\%). At the time of MLM diagnosis, six patients showed $\leq 2$ metastatic nodules, while the remaining patients showed $\geq 3$ metastatic nodules.

After MLM, 60\% patients were treated with chemotherapy, while five died before starting any treatment. Among treated patients, 12 patients performed a surgical pulmonary resection, while the remaining patients preformed only medical treatments. Regarding medical treatments, patients were treated with the following regimens: dacarbazine, BOLD + G-CSF regimen, ipilimumab and vemurafenib (for patients with B-RAF mutation). BOLD + G-CSF regimen included bleomycin at $15 \mathrm{mg}$ intravenously on day 1 and 4, Oncovin (or Vincristine) at $1 \mathrm{mg} / \mathrm{m}^{2}$ intravenously on day 1 and 5 , lomustine at 80 $\mathrm{mg} / \mathrm{m}^{2}$ on day 1 and dacarbazine at $200 \mathrm{mg} / \mathrm{m}^{2}$ intravenously on day 1 through 5, while in the intervals recombinant human G-CSF at the dosage of $5 \mu \mathrm{g} / \mathrm{kg} /$ day was administered sub cutis three times a week for 6 weeks. 
The analysis of melanoma Lung Metastases Survival (MLMS) has resulted in a median MLMS of 10 months (range 1 - 89 months).

Median Disease Free Survival (DFS) was 23 months, while median time to Melanoma Lung Metastases (TMLM) was equal to 27.4 months. Specifically, patients with a Breslow thickness $\geq 1.01 \mathrm{~mm}$ showed a median TMLM of 24 months, while for patients with Breslow thickness less than $\leq 1.0 \mathrm{~mm}$ the median TMLM was 48 months $(\mathrm{p}=0.4)$. For patients without ulceration TMLM was equal to 47 months, while for patients with ulceration was 23 months $(\mathrm{p}=0.8)$. Patients with axial primary sites showed a TMLM of 43 months while for those with peripheral sites it was 7 months $(p<0.001)$ (Figure 1a). Patients with an age $\geq 61$ years showed a TMLM of 25 months, while patients $\leq 60$ years had a TMLM of 30 months $(p=0.01)$ (Figure 1b). Finally a better behavior was observed for female patients, with a TMLM of 31 months compared to male patients $(\mathrm{p}=0.05)$.

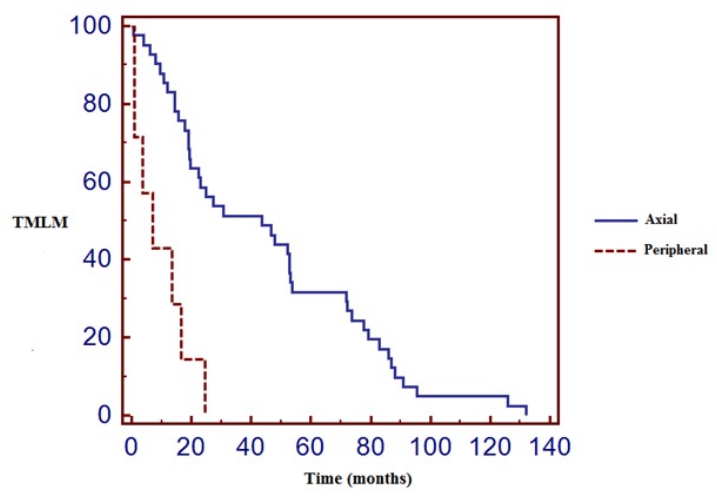

Figure 1a: Kaplan-Meier curves of Time to Melanoma Lung Metastases (TMLM) according to anatomical site of the primary tumor (axial or peripheral)

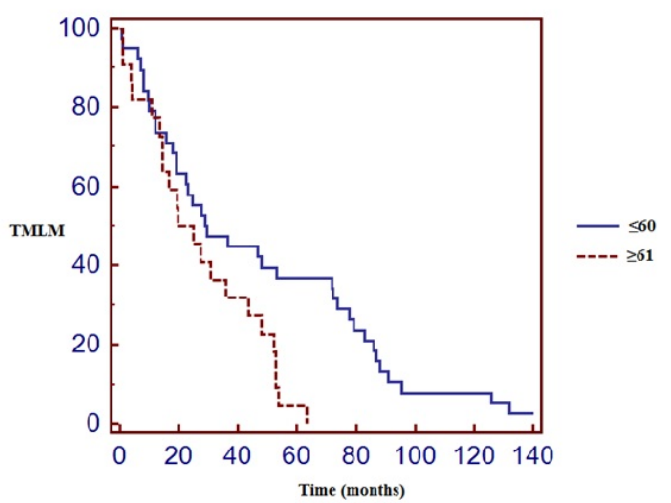

Figure 1b: Kaplan-Meier curves of Time to Melanoma Lung Metastases (TMLM) according to age ( $\leq 60$ or $\geq 61$ years)

Performing Cox proportional hazards regression between the single variables and TMLM, we found that anatomical site of the primary tumor remained the main predictor for development of MLM $(\mathrm{p}=0.01)$ (Table 2).

\begin{tabular}{|c|c|c|c|c|c|c|}
\hline & TMLM & & & os & & \\
\hline & $\begin{array}{l}\text { (median } \\
\text { months) }\end{array}$ & $p^{a}$ & $p^{b}$ & $\begin{array}{l}\text { (median } \\
\text { months) }\end{array}$ & $p^{c}$ & $p^{d}$ \\
\hline Gender & & & & & & \\
\hline Male & 23 & 0.05 & 0.7 & 50 & 0.4 & 0.5 \\
\hline Female & 31 & & & 83 & & \\
\hline Age & & & & & & \\
\hline$\geq 61$ & 25 & 0.01 & 0.2 & 35 & 0.2 & 0.4 \\
\hline$\leq 60$ & 30 & & & 83 & & \\
\hline Sites & & & & & & \\
\hline Axial & 43 & $<0.001$ & $<0.01$ & 87 & $<0.001$ & 0.04 \\
\hline Peripheral & 7 & & & 20 & & \\
\hline Ulceration & & & & & & \\
\hline Presence & 23 & 0.8 & 0.3 & 68 & 0.3 & 0.3 \\
\hline Absence & 47 & & & 46 & & \\
\hline Breslow & & & & & & \\
\hline$\geq 1.01 \mathrm{~mm}$ & 24 & 0.4 & 0.5 & 54 & 0.9 & 0.9 \\
\hline$\leq 1.00 \mathrm{~mm}$ & 48 & & & 61 & & \\
\hline SLN & & & & & & \\
\hline Positive & 29 & 0.3 & 0.9 & 35 & 0.07 & 0.08 \\
\hline Negative & 19 & & & 61 & & \\
\hline 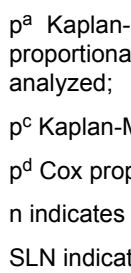 & $\begin{array}{l}\text { ier produc } \\
\text { hazards-re } \\
\text { er product } \\
\text { tional haza } \\
\text { mber of pa } \\
\text { sentinel ly }\end{array}$ & $\begin{array}{l}\text { and log-r } \\
\text { ession } \\
\text { d log-ran } \\
\text {-regress } \\
\text { ts; } \\
\text { h node. }\end{array}$ & $\begin{array}{l}\text { ak test } \\
\text { tween } \\
\text { est bety } \\
\text { h betwe }\end{array}$ & $\begin{array}{l}\text { tween TM } \\
\text { MLM and } \\
\text { en OS curv } \\
\text { OS and pr }\end{array}$ & $\begin{array}{l}\text { s; } \\
\text { dictors ane }\end{array}$ & Iyzed; \\
\hline
\end{tabular}

Table 2: Time to melanoma lung metastases (TMLM), overall survival (OS) and clinicopathological features in melanoma lung metastates

OS of the entire cohort of patients with MLM, after primary melanoma diagnosis, was 55.5 months (range 1.3-189 months). We analyzed OS of MM patients with MLM using Kaplan-Meier product according to data previously described: gender, age, sites of primary tumor, Breslow thickness, ulceration and SLN. Patients with negative SLN, without ulceration, with a Breslow thickness $\leq 1.00 \mathrm{~mm}$, and female patients showed a better OS; however only axial site reached a statistical significance $(\mathrm{p}<0.04)$ (Figure 2$)$. Also in the long-term, at Cox proportional hazards regression, anatomical site remained the main predictor $(\mathrm{p}=0.04)$ (Table 2$)$. 


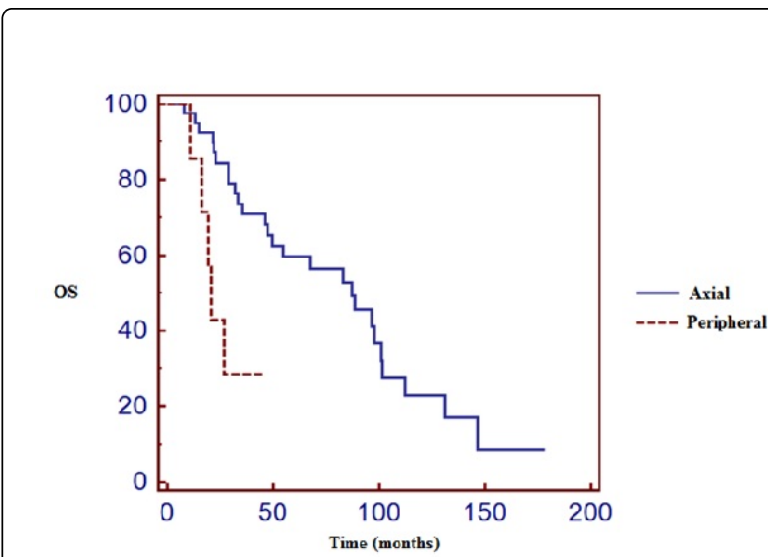

Figure 2: Kaplan-Meier curves of overall survival (OS) of melanoma lung metastases (MLM) according to anatomical site of the primary tumor ( axial or peripheral)

\section{Discussion}

According to 2009 American Joint Cancer Committee Staging Melanoma Database, patients with melanoma lung metastases (MLM) are classified as IV M1b, with a 1-year survival rate of 51\% [2]. In literature, there are several reports about surgical approaches to MLM, while there are few data regarding clinicopathological predictors to develop MLM. Like Balch et al. [3], we included in the current report patients who performed pulmonary metastasectomy as well as patients who were not considerated to be surgical candidates. To date, metastasectomy remains the primary treatment for lung metastases with $\leq 2$ nodules, while anti-CTL4 antibody (ipilimumab), anti-PD1 antibody (nivolumab), vemurafenib and the association between dabrafenib and trametenib (for B-RAF mutations), are the most recent chemotherapy treatments, which have shown an excellent therapeutical response in this class of patients. However, the aim of our study was not to evaluate the surgical and/or the medical approach to MLM, but rather to evaluate the predictors of the primary tumor to develop MLM.

Reviewing the literature and analyzing 1.105 MLM patients from 8 different reports, we found that, after a complete resection of MLM, the median melanoma lung metastases survival (MLMS) was equal to 25 months, compared to a median of 11 months in the unresectable ones [4-11]. Our general MLMS was of 10 months, approaching the data usually reported regarding unresectable and multiple MLM [4]. In fact, probably, the high percentage of multiple MLM in the current report could explain the low median MLMS found in our analysis. In this regard, the importance of the number of MLM has been emphasized by many authors, with a high survival rate for patients with $\leq 2$ lesions [4-12].

Also regarding TMLM, we found a median TMLM comparable with the one previously reported [6]. In this regard, according to our results and to the literature, the fact that median TMLM of all MLM occurred before 4 years, highlights the importance of a strict follow-up in the early time period, following the diagnosis of the primary melanoma, in order to reduce the risk of pulmonary recurrences, and to improve and extend TMLM.

As reported in literature, in our study the lung represented the first metastatic site in the majority of patients (62\%). Besides, regarding patients who performed a sentinel lymph node biopsy (SLN), excluding melanoma with unknown primary, only $17 \%$ of patients presented a positive SLN, while the remaining were all negative, and in two patients SLN was not performed because not provided (pTIA, AJCC stage IA). In fact, in a previous report, analyzing 292 patients with MLM, only 36 patients (12.3\%) had positive nodes [5]. The high percentage of extra-nodal region as first metastatic sites and the low percentage of nodal involvement in MLM patients show that the conventional metastatic nodal dissemination [12] has not followed. This percentage may indicate that the number of skip metastases in this class of patients is higher than the average percentage detected in general melanoma population, where skip metastases account to $\leq 1 \%$ [13]. Up to now, the influence of lymph nodal involvement at the time of MLM remains unclear and require further investigations [5,7].

Regarding the first anatomical site, it is known that many of the preferences observed for the spread of specific cancers to specific metastatic locations can be explained by the direction of blood flow. In this regard, organs in close proximity are likely to be the main sites of metastases for a particular primary tumor. While, generally, malignant melanoma is usually considered as different from other solid tumors, because it is a cutaneous malignancy with straightforward lymphatic flow, which can be mapped [7]. However (as reported above) about one-third of the locations of frequent metastases is puzzling about this observation. In our sample, although $68 \%$ of patients showed an axial primary tumor, TMLM $(\mathrm{p}<0.001)$ and OS $(\mathrm{p}<0.001)$ were both worse for those arisen in the peripheral sites. At the Cox proportional hazards-regression, anatomical site remained the main predictor.

Conversely the literature, where ulceration is a marker of an increased risk of hematologic dissemination, extracellular matrix invasion and subsequent development of distant metastases (independently of tumor thickness), ulceration did not remain a predictor in the statistical analysis, as observed in case of melanoma brain metastases (although we found a worse TMLM and OS for ulcerated melanoma) $[14,15]$. These results, in contrast with Reintgen et al. [13], highlight that probably the route of lung metastatization in MLM does not follow the conventional pathophysiological processes involved and widely known for melanoma. This observation is confirmed also by the low percentage of nodal involvement in our sample. In this regard, the presence of a malignant melanoma of the peripheral sites is associated with a higher risk of MLM than the ones arose in the axial sites, regardless of the other clinicopathological aspects.

Despite the advent of new therapeutic treatments, mortality rate due to melanoma continues to rise at an alarming rate. Our report suggested that after the surgical removing of the primary tumor (also in absence of metastatic recurrences), according to clinicopathologic predictors, it may be selected a class of melanoma patients with an increased risk of MLM, in order to perform a close clinical and instrumental follow-up and/or to perform a tertiary chemoprevention (identifying target patients who have had previous melanoma, in order to prevent development of secondary recurrences), since currently there are no approved adjuvants therapies for this malignancy. In this regard, the prevention of secondary recurrences (especially in melanoma-target organs, such as lungs) plays a pivotal role in the management of melanoma. Therefore, identify target patients in order 
Citation: Paolino G, Bottoni U, Clerico R, Didona D, Venuta F, et al. (2014) Clinicopathological Predictive Factors of Melanoma Lung Metastases. J Integr Oncol 3: 119. doi:10.4172/2329-6771.1000119

Page 5 of 5

to avoid "target organ recurrences" (as MLM) remains a crucial point in the management of malignant melanoma, as well as for the other malignancies.

\section{Acknowledgements}

The manuscript was supported by Associazione Romana Ricerca Dermatologica.

\section{References}

1. Mohammed TL, Chowdhry A, Reddy GP, Amorosa JK, Brown K, et al. (2011) ACR Appropriateness Criteria ${ }^{\circ}$ screening for pulmonary metastases. J Thorac Imaging 26: W1-3.

2. Balch CM1, Gershenwald JE, Soong SJ, Thompson JF, Atkins MB, et al. (2009) Final version of 2009 AJCC melanoma staging and classification. J Clin Oncol 27: 6199-6206.

3. Balch CM, Soong S, Ross MI, Urist MM, Karakousis CP, et al. (2000) Long-term results of a multi-institutional randomized trial comparing prognostic factors and surgical results for intermediate thickness melanomas (1.0 to $4.0 \mathrm{~mm}$ ). Intergroup Melanoma Surgical Trial. Ann Surg Onco 7: 87-97.

4. Neuman HB, Patel A, Hanlon C, Wolchok JD, Houghton AN, et al. (2007) Stage-IV melanoma and pulmonary metastases: factors predictive of survival. Ann Surg Oncol 14: 2847-2853.

5. Chua TC, Scolyer RA, Kennedy CW, Yan TD, McCaughan BC, et al. (2012) Surgical management of melanoma lung metastasis: an analysis of survival outcomes in 292 consecutive patients. Ann Surg Oncol 19: 1774-1781.

6. Andrews S, Robinson L, Cantor A, DeConti RC (2006) Survival after surgical resection of isolated pulmonary metastases from malignant melanoma. Cancer Control 13: 218-223.
7. Leo F, Cagini L, Rocmans P, Cappello M, Geel AN, et al. (2000) Lung metastases from melanoma: when is surgical treatment warranted? $\mathrm{Br} \mathrm{J}$ Cancer 83: 569-572.

8. Oliaro A, Filosso PL, Bruna MC, Mossetti C, Ruffini E (2010) Pulmonary metastasectomy for melanoma. J Thorac Oncol 5: S187-191.

9. Petersen RP, Hanish SI, Haney JC, Miller CC 3rd, Burfeind WR Jr, et al. (2007) Improved survival with pulmonary metastasectomy: an analysis of 1720 patients with pulmonary metastatic melanoma. J Thorac Cardiovasc Surg 133: 104-110.

10. Ollila DW, Stern SL, Morton DL (1998) Tumor doubling time: a selection factor for pulmonary resection of metastatic melanoma. J Surg Oncol 69: 206-211.

11. Tafra L, Dale PS, Wanek LA, Ramming KP, Morton DL (1995) Resection and adjuvant immunotherapy for melanoma metastatic to the lung and thorax. J Thorac Cardiovasc Surg 110: 119-128.

12. Slingluff CL Jr, Vollmer RT, Reintgen DS, Seigler HF (1988) Lethal "thin" malignant melanoma. Identifying patients at risk. Ann Surg 208: 150-161.

13. Reintgen D, Cruse CW, Wells K, Berman C, Fenske N, et al. (1994) The orderly progression of melanoma nodal metastases. Ann Surg 220: 759-767.

14. Zakrzewski J, Geraghty LN, Rose AE, Christos PJ, Mazumdar M, et al. (2011) Clinical variables and primary tumor characteristics predictive of the development of melanoma brain metastases and post-brain metastases survival. Cancer 117: 1711-1720.

15. Bottoni U, Clerico R, Paolino G, Ambrifi M, Corsetti P, et al. (2013) Predictors and survival in patients with melanoma brain metastases. Med Oncol 30: 466 